\title{
SOP Periprothetische Femurfraktur
}

Michael Kremer, Reinhard Hoffmann

\section{Einleitung}

Aufgrund der Kombination von steigender Lebenserwartung, anhaltender Aktivität bis ins hohe Lebensalter sowie einer steigenden Zahl an Prothesenimplantationen (erwarteter Anstieg um 174\% bis 2030) ist von einem überproportionalem Anstieg an periprothetischen Frakturen in Zukunft auszugehen. So zeigen Registerdaten in den letzten zehn Jahren bereits mehr als eine Verdopplung der Inzidenz.

- Die Inzidenz periprothetischer Frakturen nach primärer Knie-TEP wird mit 0,3-5,5\% angegeben, nach Prothesenwechsel mit bis zu 35\%. Über $80 \%$ der Frakturen betreffen das distale Femur, seltener treten Frakturen an der proximalen Tibia und der Patella auf [5].

- Bei primärer Hüft-TEP wird die Inzidenz periprothetischer Frakturen mit 1-4\% angegeben, nach Revisionseingriffen mit bis zu $17 \%$. Auch hier ist vor allem das Femur betroffen, Beckenfrakturen bei implantierter Hüftpfanne sind deutlich seltener.

Es zeigen sich aufgrund unterschiedlicher Pathologien zwei typische Altersgruppen für diese Frakturen, zum einen jüngere Patienten unter 60 Jahre und Patienten über 80 Jahre. Bei den jüngeren, zumeist knochengesunden Patienten werden typischerweise adäquate Unfallhergänge, häufig Hochrasanzverletzungen, beschrieben. Frakturen als Folge von Osteolysen oder Prothesenlockerungen werden seltener beobachtet. Gerade bei diesen Patienten muss besonders auf begleitende Weichteilverletzungen und Kombinationsverletzungen geachtet werden.

Bei den älteren Patienten liegt meist eine Kombination aus schlechter Knochenqualität und Prothesenlockerung vor. Zusätzlich besteht häufig eine erhöhte Sturzneigung, die zu einem deutlichen Risikoanstieg für periprothetische Frakturen führt. In dieser Altersgruppe treten ca. 75\% der postoperativen Frakturen als Folge niederenergetischer Verletzungen bzw. ganz ohne adäquates Trauma auf. Entsprechend sind das begleitende Weichteiltrauma und die Rate an Begleitverletzungen zumeist deutlich geringer.
Risikofaktoren für periprothetische Frakturen:

- Patientenalter

- verminderte Knochenqualität:

- Osteoporose, Osteomalazie, Morbus Paget

- rheumatische Erkrankungen, Steroidmedikation

- Stress Shielding nach Prothesenimplantation

- mechanische Knochenschwächung:

- lokale Osteolyse, alte Frakturen, Schraubenlöcher, femorales Notching etc.

- septische/aseptische Lockerung

- erhöhte Sturzneigung: Schwindel, Morbus Parkinson, Epilepsie, Ataxie, Synkopen, Gangunsicherheit

Diese kurze SOP ist nicht geeignet, um eine differenzierte Behandlung der zum Teil sehr komplexen und individuellen Behandlung von periprothetischen Frakturen zu ermöglichen. Sie soll jedoch einen Überblick über die Behandlungsoptionen sowie das grundlegende Management dieser Frakturen geben.

\section{Präoperative Diagnostik und Klassifikation}

\section{Anamnese}

Eine umfassende Anamnese, insbesondere auch der internistischen Grunderkrankungen, ist bei den häufig multimorbiden Patienten unerlässlich. Besonderes Augenmerk sollte z. B. auf einer bestehenden Antikoagulation (insbesondere NOAK), vorbestehenden neurologischen Defizite sowie dem funktionellen Anspruch liegen.

Zudem ist auch eine differenzierte Erhebung der Unfallanamnese wesentlich. Insbesondere die Unterscheidung zwischen hoch- und niederenergetischen Unfällen ist in Hinblick auf relevante begleitende Weichteilverletzungen richtungsweisend.

Die Erhebung der „Prothesenanamnese“ ist wesentlich zur Entscheidung, ob ein Prothesenerhalt möglich und sinnvoll ist oder ein Wechsel notwendig sein kann [9]:

- Prothesenmodell (OP-Bericht oder Implantatpass), Implantationsdatum

- postoperativer Verlauf (Zeichen für Infekt, Lockerung etc.)

- ggf. Voraufnahmen im Verlauf bei V.a. Sinterung oder Migration 

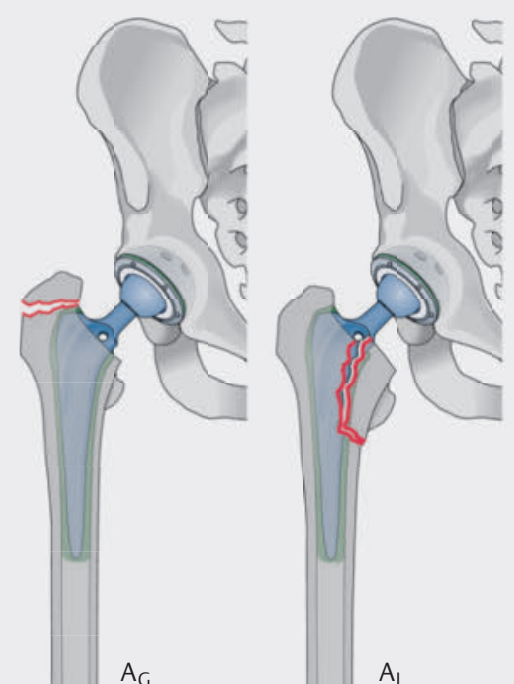

$A_{L}$

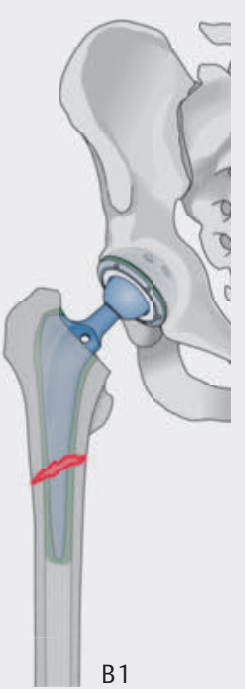

B1
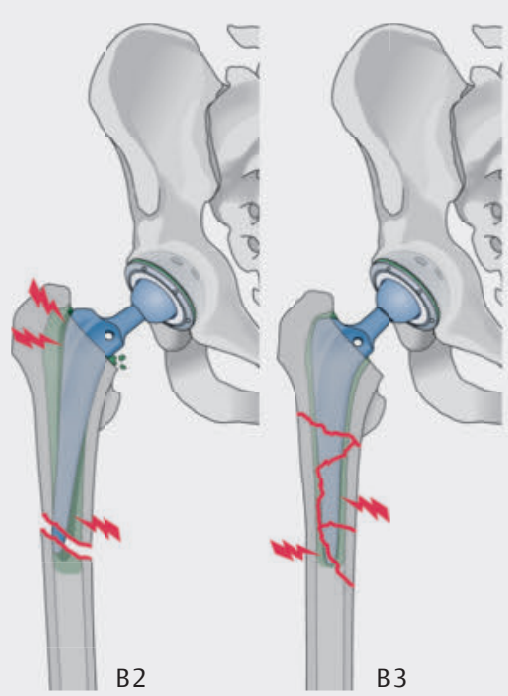

- Abb. 1 Vancouver-Klassifikation. Klassifikation periprothetischer Frakturen nach Hüft-TEP. (Quelle: Biber R, Bail H. Frakturen im Alter. Orthopädie und Unfallchirurgie up2date 2014; 9: 207-223)

- Funktion und Zufriedenheit vor der Fraktur (Belastungsschmerz, eingeschränkte Gehstrecke, Instabilität)

\section{Klinische Untersuchung}

- vollständiger Bodycheck, insbesondere bei Hochrasanzverletzungen

- Untersuchung des betroffenen Gelenks (soweit schmerzbedingt möglich)

- immer gesamte Extremität untersuchen (Kettenverletzung, weitere Implantate etc.)

- periphere Durchblutung, Motorik, Sensibilität, ggf. neurologisches Konsil

- Beurteilung des Haut- und Weichteilverhältnisse

- vorbestehende Zugänge (Planung der Revisions-OP)

\section{Bildgebende Untersuchung}

Radiologische Standarddiagnostik:

- betroffenes Gelenk in zwei Ebenen

- (ggf.) Patella-Tangentialaufnahme

- Eine vollständige Abbildung sämtlicher Prothesenkomponenten ist unerlässlich.

- Ober-/Unterschenkel-Ganzaufnahme (weitere Prothese, Frakturausläufer, Kettenverletzung)

- Eine Computertomografie ist insbesondere bei komplexen Frakturformen, aber auch bei fraglichen Lockerungen, Lysen etc. zur präoperativen Planung indiziert.

- Weitere diagnostische Verfahren (MRT, Knochenszintigrafie etc.) sind regelhaft nicht erforderlich.
Merke

Radiologische Kriterien, die für eine Prothesen-

lockerung sprechen [7]:

- Lysesaum im Bereich der Prothese

- Sinterung/Lageänderung der Prothese

- Zementseparation

- Frakturausläufer bis zum Implantat

\section{Klassifikation}

Eine adäquate Klassifikation der Fraktur ist unerlässlich, da die gebräuchlichen Klassifikationen als Entscheidungsgrundlage für die weitere Therapie (Osteosynthese, Prothesenwechsel etc.) dienen können.

\section{Klassifikation periprothetischer Frakturen nach Hüft-TEP (Vancouver-Klassifikation)}

Die verbreitetste Klassifikation für periprothetische Frakturen am Hüftgelenk ist die Vancouver-Klassifikation [8]. Diese berücksichtigt die Frakturlokalisation, Prothesenstabilität und Knochenqualität und ist hilfreich für die Planung der weiteren Versorgung.

Es werden drei Hauptfrakturtypen unterschieden

Abb. 1):

- Typ-A-Frakturen beschreiben Trochanterfrakturen, wobei Typ AG Frakturen des Trochanter major, Typ AL diejenigen des Trochanter minor beschreibt.

- Typ-B-Frakturen stellen mit ca. 80\% die häufigsten Frakturen dar und sind im Bereich des Prothesenschafts lokalisiert. Hier wird zwischen stabiler Prothese (B1), gelockerter Prothese (B2) und gelockerter Prothese bei schlechter Knochenqualität (B3) unterschieden. 
- Typ-C-Frakturen sind Frakturen unterhalb des Prothesenstiels bei stabilem Implantat.

\section{Klassifikation periprothetischer Frakturen} nach Knie-TEP (Lewis-Rorabeck-Klassifikation)

Periprothetische Frakturen nach Knie-TEP können z.B. nach Lewis-Rorabeck klassifiziert werden. Entscheidende Parameter dieser Klassifikation sind der Dislokationsgrad der Fraktur sowie die Stabilität der Prothese. Das Frakturmuster wird in dieser pragmatischen Klassifikation anders als z.B. in der auch gebräuchlichen Klassifikation nach Su nicht berücksichtigt. Trotzdem lassen sich die wesentlichen Therapieoptionen aus dieser Klassifikation herleiten ( $\boldsymbol{A} \mathbf{A b b} . \mathbf{2})$ :

- Typ I: nichtdislozierte Fraktur bei nicht gelockerter Prothese

- Typ II: dislozierte Fraktur bei nicht gelockerter Prothese

- Typ III: dislozierte oder nicht dislozierte Fraktur bei gelockerter Prothese

Die selteneren Patellafrakturen (ca. 0,7\% aller Frakturen) können z.B. nach Goldberg, die periprothetischen Tibiafrakturen (ca. $4 \%$ aller Frakturen) z. B. nach Felix eingeteilt werden. Aufgrund der niedrigen Inzidenz wird in dieser SOP auf eine detaillierte Darstellung verzichtet.

\section{UCS-Klassifikation}

Ein Versuch, eine einheitliche Klassifikation für alle Arten der periprothetischen Frakturen zu erstellen, ist das Unified Classification System (UCS) von Duncan und Haddad [2]. Hier wird in sechs Haupttypen (Typ A-F) jede mögliche Frakturform um eine oder mehrere Prothesen sowie in einem oder beiden gelenkbildenden Knochen beschrieben. Die Typen A-C sind analog zur VancouverKlassifikation. Zusätzlich sind noch die Typen D-F eingeführt worden:

- Typ D sind Brüche eines Knochens zwischen zwei Prothesen (interprothetische Fraktur), z. B. Femurschaft nach Hüft- und Knie-TEP.

- Typ E beschreibt Brüche von zwei Knochen um eine Prothese (z. B. Femur und Tibia bei liegender KnieTEP).

- Typ F sind die seltenen Fälle mit Brüchen einer nicht ersetzten Gelenkfläche bei Teilgelenkersatz (z. B. Azetabulumfraktur bei liege > nder Duokopfprothese)

\section{Indikationsstellung} und Verfahrenswahl

Für die operative Therapie ist eine differenzierte Planung essenziell. Zur Verfahrenswahl müssen vor allem Frakturtyp, Prothesenstabilität sowie die Knochenqualität analysiert werden. Zudem sind Faktoren wie vorhergehende Funktion des Gelenks, Verankerungsmöglichkeiten der Osteosynthese bzw. Revisionsprothese, Anspruch des Pa-

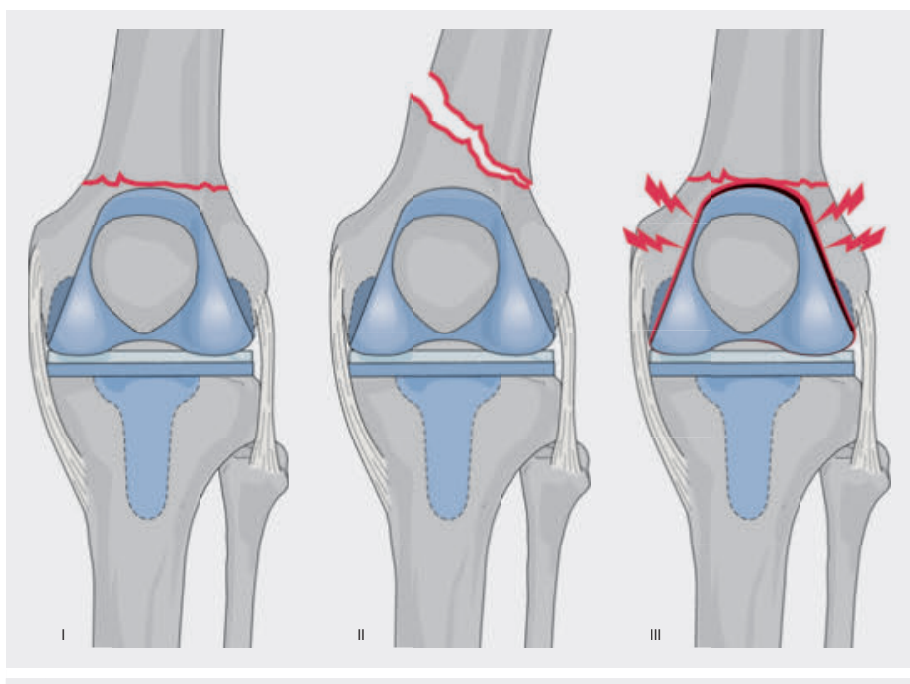

- Abb. 2 Lewis-Rorabeck-Klassifikation. Klassifikation periprothetischer Frakturen nach Knie-TEP. (Quelle: Biber R, Bail H. Frakturen im Alter. Orthopädie und Unfallchirurgie up2date 2014; 9: 207-223)

tienten, Operationsrisiko, Compliance etc. mit zu bewerten. Auch wenn periprothetische Frakturen eine differenzierte und häufig auch individuelle Therapieplanung bedürfen, orientiert sich die Therapie in der Regel an den oben genannten Klassifikationen.

\section{Konservative Therapie}

Die konservative Therapie zeigt eine hohe Rate an späteren operativen Revisionen und ein schlechtes funktionelles Ergebnis bedingt durch die notwendige lange Ruhigstellung. Auch bei nichtdislozierten Frakturen wird aufgrund der langen Immobilisationsdauer und des hohen Risikos einer sekundären Dislokation unter konservativer Therapie heutzutage in der Regel die operative Versorgung empfohlen $[4,10]$. Bis auf wenige Ausnahmen sind periprothetische Frakturen daher eine Domäne der operativen Therapie.

\section{Therapie periprothetischer Frakturen nach Hüft-TEP}

Typ-A-Frakturen

Typ-A-Frakturen sind solche im Bereich des Trochanter major (AG) bzw. Trochanter minor (AL). Um mehr als $2 \mathrm{~cm}$ dislozierte Trochanter-major-Frakturen sollten zur Vermeidung einer pelvitrochantären Insuffizienz wenn möglich refixiert werden. Hier sind Zerklagen und diverse Plattenosteosynthesen beschrieben. Die Komplikationsraten (sekundäre Dislokation, Pseudarthrose, störendes Implantat etc.) sind jedoch hoch. Häufig verbleiben trotz Operation funktionelle Einschränkungen.

Typ-B-Frakturen

Typ-B-Frakturen liegen im Schaftbereich und werden in Abhängigkeit von der Stabilität der Prothese sowie der Knochenqualität weiter unterteilt ( $\bullet$ Abb. 3): 

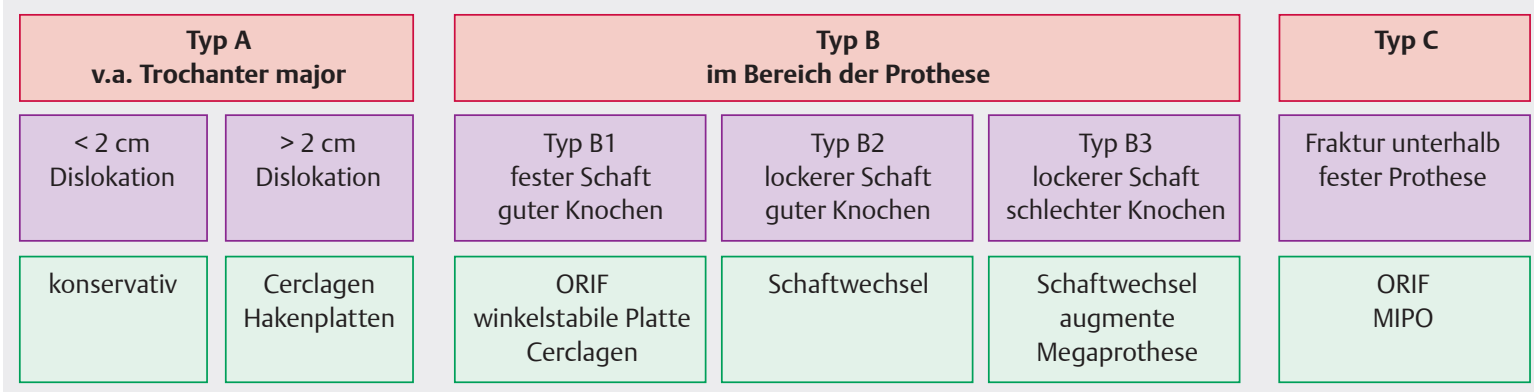

- Abb. 3 Periprothetische Fraktur nach Hüft-TEP. Behandlungsschema.

- Bei B1-Frakturen ist die Prothese stabil. Die Standardversorgung ist die Plattenosteosynthese über einen lateralen Zugang, der nach proximal und distal erweiterbar ist. Hier stehen diverse winkelstabile Implantatsysteme zur offenen oder minimalinvasiven Versorgung zur Verfügung. Es sollte versucht werden, zumindest 8-10 Kortizes im proximalen und distalen Hauptfragment zu verankern. Es gibt spezielle periprothetische Plattensysteme mit versetzten Lochreihen, die eine Schraubenplatzierung an der Prothese vorbei vereinfachen. Alternativ ist z. B. eine Standardplatte mit zusätzlicher LAP (Locking Attachement Plate) bzw. die Verwendung von Zerklagen eine sinnvolle Option. Durch die Verwendung winkelstabiler Plattenosteosynthesen konnte die Rate an Pseudarthrosen und Implantatversagen von fast 50\% auf etwa $5 \%$ reduziert werden.

- B2-Frakturen mit gelockertem Schaft und guter Knochenqualität benötigen typischerweise einen Schaftwechsel. Hier haben sich in Europa modulare oder nichtmodulare, distal verankernde konische Revisionsprothesen etabliert. Sowohl modulare als auch nichtmodulare Prothesen haben spezifische Vor- und Nachteile, die bei der Indikationsstellung beachtet werden müssen. Entscheidend ist die ausreichend lange Verankerungsstrecke im Isthmus, die etwa $4 \mathrm{~cm}$ betragen sollte. Die zementierte Versorgung zeigt schlechtere Ergebnisse und ist als Standardverfahren nicht empfohlen. In ausgewählten Situationen kann ein zementiertes Vorgehen zumindest eine Therapiealternative sein.

- Bei B3-Frakturen stellt die schlechte Knochenqualität ein wesentliches Problem in der Behandlung dar. Ist noch ein ausreichend stabiler Isthmus vorhanden, kann analog der B2-Frakturen vorgegangen werden. Bei instabilem Isthmus ist ein modularer, distal zementierbarer Schaft eine Option, evt. auch ein distal verriegelbarer zementfreier modularer Revisionsschaft. Bei beiden Verfahren muss die distale Knochenqualität jedoch eine stabile Verankerung zulassen. Als Alternative kann ein intramedullärer Femurersatz mit entsprechend größerem Operationstrauma erwogen werden. Auch eine knöcherne Augmentation (Spon- giosa, Strutgraft, Allograft) wird mit z.T. sehr guten Ergebnissen verwendet und ist bei defizitärem Knochen eine zusätzlich Option.

\section{Typ-C-Frakturen}

Typ-C-Frakturen sind Brüche in der Nähe von Prothesen, ohne diese jedoch zu erreichen. Lockerungen der Prothese liegen klassischerweise nicht vor; sie werden analog zu den B1-Frakturen osteosynthetisch mittels winkelstabiler Plattenosteosynthese versorgt.

\section{Therapie periprothetischer Frakturen nach Knie-TEP}

Bei den periprothetischen distalen Femurfrakturen ist vor allem die Stabilität der Prothese entscheidend. RorabeckTyp-1- und -2-Frakturen mit stabiler Prothese werden wenn immer möglich osteosynthetisch versorgt. Neben der winkelstabilen Plattenosteosynthese über einen minimalinvasiven oder konventionellen lateralen Zugang kann bei „Open-Box“-Prothesen auch ein retrograder Marknagel verwendet werden. Hierzu ist die Kenntnis des Implantats mit Durchmesser der Box bzw. ein CT zur Messung der Boxgröße sowie zur Planung der späteren Nagellage zwingend erforderlich. Die Literatur zeigt jedoch bessere Ergebnisse für die Plattenosteosynthese, so dass diese zu bevorzugen ist. Wenn möglich sollte diese in weichteilschonender, minimalinvasiver Technik durchgeführt werden.

Die Notwendigkeit zum Prothesenwechsel bei stabilem femoralem Schild ist die Ausnahme. Hier sind insbesondere sehr distale, bis weit unter die Prothese reichende Frakturen kritisch. Zum einen liegt doch häufig eine Lockerung der Komponenten vor. Zudem ist bei unzureichender Verankerung im gelenktragenden Fragment eine Osteosynthese nicht möglich, und zumeist bleibt nur der Kondylen-bzw. distale Femurersatz. Insbesondere bei diesen Frakturformen muss eine Revisionsprothese vorgehalten werden.

Bei den Rorabeck-Typ-3-Frakturen ist die Prothese gelockert und ein Prothesenwechsel notwendig. Typischerweise werden hier langstielige Prothesen verwendet, die 
den Frakturbereich überbrücken, meist kombiniert mit einer Plattenosteosynthese je nach Lokalisation der Fraktur. In Abhängigkeit vom knöchernen Defekt kann auch hier ein Kondylenersatz oder distaler Femurersatz notwendig werden und ist vorzuhalten.

Wie bei allen periprothetischen Frakturen am Knie ist auch in diesen Situationen ein funktionsfähiges Gelenk das Ziel. Liegt zusätzlich aber ein insuffizienter und nicht rekonstruierbarer Streckapparat vor, ist die Arthrodese als Therapieoption kritisch zu diskutieren. Die Amputation ist bei den meist älteren Patienten nur als Ultima Ratio zu sehen, da hiernach häufig eine Bettlägerigkeit und entsprechende Pflegebedürftigkeit zu erwarten sind.

\section{Therapie periprothetischer Tibia- bzw. Patellafrakturen}

Diese Frakturen sind sehr selten und sollen daher an dieser Stelle nur kurz erwähnt werden. Die meisten Frakturen der proximalen Tibia (ca. 80\%) sind Folge von Implantatlockerungen und werden mit einem Komponentenwechsel behandelt. Auch hier können langstielige Schäfte und knöcherne oder metallische Augmente (z. B. Tantaloder Titan-Cones) verwendet werden.

Bei größeren Defekten kann ein proximaler Tibiaersatz notwendig werden. Hier ist jedoch die Refixation des Streckapparats ein relevantes Problem. Letztlich führt eine insuffiziente Einheilung zu einem Funktionsverlust des Streckapparats und damit des gesamten Kniegelenks.

Frakturen bei stabilem Tibiaplateau werden osteosynthetisch durch Schrauben- bzw. Plattenosteosynthesen versorgt. Tuberositas-tibiae-Ausrisse werden typischerweise verschraubt oder mittels Hakenplatte versorgt.

Patellafrakturen liegt häufig eine Kombination aus femoralem Overstuffing, geringer Patelladicke und (Teil-) Nekrosen zugrunde. Seltener sind adäquate Traumen mit direktem Sturz auf die Patella ursächlich. Die Versorgung dieser Frakturen ist häufig schwierig. Wenn möglich, sollten winkelstabile Plattenosteosynthesen oder alternativ kanülierte Zugschraubenosteosynthesen mit zusätzlicher Tonnenzerklage zur Anwendung kommen.

\section{Operationszeitpunkt}

Patienten mit periprothetischen Femurfrakturen haben ein ähnliches Risikoprofil wie die proximalen Femurfrakturen (ca. $3 / 4$ der Patienten sind ASA III-V). Vergleichbar zu den Schenkelhalsfrakturen erhöht sich die Mortalität nach periprothetischen Frakturen signifikant bei Versorgungen später als 48 Stunden nach Trauma. Ebenfalls vergleichbar ist die Ein-Jahres-Letalität mit ca. 17-24\%.

Trotz dem Wunsch nach möglichst zeitnaher Versorgung sind optimale Voraussetzungen hinsichtlich der Narkose
(z.B. Einnahme von NOAK, Marcumar, präoperative Intensivtherapie), des OP-Teams sowie des Vorhandenseins benötigter Implantate und Rückzugsoptionen (Komponentenwechsel, Revisionssysteme etc.) zwingend.

Die Ruhigstellung bis zur Operation erfolgt in einer Kastenschiene. Eine Drahtextension sollte nur im begründeten Ausnahmefall angewendet werden, wenn eine einfache Ruhigstellung nicht ausreichend (grobe Dislokation, Fragmentdruck etc.) und keine zeitnahe operative Versorgung möglich ist.

\section{Aufklärung und Komplikationen}

Die wesentlichen Punkte der Aufklärung sind der folgenden Checkliste zu entnehmen. Eine konservative Therapie ist nur bei ausgeprägtem OP-Risiko und nichtdislozierter Fraktur zu erwägen. Ansonsten zeigen sich regelhaft schlechtere Ergebnisse im Vergleich zur operativen Therapie.

\section{Allgemeine Operationsrisiken}

Allergien, Thrombose, Lungenembolie, Fettembolie, Verletzung von benachbarten Strukturen (Gefäße/Nerven/ Sehnen/Muskeln), Blutung, Nachblutung, Infektion, Wundheilungsstörung, Lagerungsschäden, Folgeoperation, Immobilität, Schmerzen.

\section{Spezielle Operationsrisiken}

- bei Osteosynthese:

- Erläuterung des operativen Vorgehens, z. B. Reposition, Osteosynthese, ggf. Spongiosaplastik/Verwendung von allogenem Knochenmaterial

- Achs-/Rotationsfehlstellung, Beinlängendifferenz

- Pseudarthrose, Implantatversagen

- immer auch über die evtl. Notwendigkeit eines ungeplanten Prothesenwechsels aufklären

- bei Prothesenwechsel:

- erhöhte Komplikationsrate im Vergleich zur Primärprothese

- intraoperative Fraktur, Prothesenluxation, -lockerung, Sinterung, schlechtere Funktion im Vergleich zur primären Prothese

- Beinlängendifferenz, muskuläre Defizite (Trendelenburg, Einschränkung Streckapparat)

- zementfreies oder zementiertes Vorgehen, „Zementreaktion“

- großzügige Aufklärung über OP-Ausweitung bis zum distalen/proximalen Femurersatz, proximaler Tibiaersatz, intramedullärer Femurersatz, Arthrodese etc.

- bei intraoperativem Infektverdacht auch zweizeitiges Vorgehen möglich mit Explantation der Prothese und Gewebeentnahme zur mikrobiologischen Analyse

- Zeitraum bis zur vollständigen Rehabilitation mindestens acht bis zwölf Wochen 
- Je nach Versorgung ist eine schmerzadaptierte Vollbelastung möglich, nach Osteosynthesen in der Regeln nur eine Teilbelastung.

\section{Präoperative Checkliste}

Neben den allgemeinen präoperativen Vorbereitungen sind folgende Punkte vor der Versorgung von periprothetischen Frakturen unbedingt zu beachten:

- Ist die Prothesenanamnese vollständig?

- OP-Bericht vorhanden/Implantate bekannt?

- evtl. notwendige Komponenten(z. B. Inlays) oder Revisionssysteme bestellt?

- bei Osteosynthese auch auf unerwartete Prothesenlockerung und notwenigen Wechsel vorbereitet sein

- Vorhandensein der erforderlichen Röntgenaufnahmen und ggf. zusätzliche Bildgebung

- präoperative (Größen-)Planung der Implantate anhand der Röntgenbilder (Messkugel verwenden!)

- Reevaluation des Allgemeinzustands des Patienten

- Kontrolle der laborchemischen Befunde und Bereitstellung der benötigten Erythrozytenkonzentrate

- Markierung der verletzten Seite

\section{Perioperative Maßnahmen}

- Operation in Intubationsnarkose

- Lagerung des Patienten in Rücken- oder Seitenlage je nach geplantem Zugang

- perioperative Single-Shot-Antibiose (z.B. Cefuroxim $1,5 \mathrm{~g}$ i. v. beim Erwachsenen)

- Rasur des OP-Gebiets

- Sicherstellung der Durchleuchtungsmöglichkeiten

- steriles Abwaschen und Abdecken des gesamten Beines (mögliche intraoperative Ausweitung des Zugangs, Möglichkeit des Prothesenwechsel)

- Instrumentarium: Grundsieb fü $r$ Knochen- und Weichteileingriffe, Repositionszangen, ggf. Prothesenwechsel-Sieb, (Kabel-)Zerklagen

- Systemsiebe in Abhängigkeit von der Fraktur und geplanten Versorgung

- monopolarer Elektrokauter, Sauger (großzügier CellSaver-Einsatz) und Saugersack, Redon-Drainagen

- Standard-Team-Time-out nach WHO-Richtlinie

\section{Postoperative Maßnahmen und Nachsorge}

Die Nachbehandlung richtet sich nach der Stabilität der Osteosynthese bzw. Prothese. Nach Prothesenwechsel ist meist eine Voll- oder zumindest Teilbelastung mit halbem Körpergewicht möglich, nach osteosynthetischer Versorgung typischerweise Sohlenkontakt oder $20 \mathrm{~kg}$ Teilbelastung.
- gipsfreie Nachbehandlung, ggf. vorrübergehende Lagerung des Beines in einer Kastenschiene

- physikalische (AV-Pumpe) und medikamentöse Thromboseprophylaxe ab sechs Stunden postoperativ

- adäquate Schmerztherapie

- Entfernung der Drainagen nach 24-48 Stunden

- Röntgen der Hüfte in zwei Ebenen am zweiten postoperativen Tag

- regelmäßige Wundkontrollen und Verbandswechsel

- physiotherapeutische Übungsbehandlung, Lymphdrainage

- bei Knierevisionen zusätzlich Motorschiene

- zunehmend aktive Beü bung nach Maßgabe der Belastbarkeit und Weichteilsituation

Da diesen Frakturen häufig eine Osteoporose zugrunde liegt, sollte postoperativ mit einer Osteoporosetherapie (Vitamin-D-Substitution) oder zumindest einer Diagnostik begonnen werden [1].

Bei den meist älteren Patienten ist frühzeitig ein Sozialdienst (Anschlussheilbehandlung, Kurzzeitpflege oder häusliche Versorgung) einzubinden und die Versorgung mit Hilfsmitteln wie Toilettensitzerhöhung, Keilkissen Greifzange einzuleiten. Nur ca. 70\% der Patienten erreichen ihr vorheriges Mobilisationsniveau, und ca. 15\% der Patienten müssen erstmalig in ein Pflegeheim.

\section{Autorinnen/Autoren}

\section{Michael Kremer}

Dr. med., Abteilung für Unfallchirurgie und Orthopädische Chirurgie, Berufsgenossenschaftliche Unfallklinik Frankfurt am Main

\section{Reinhard Hoffmann}

Prof. Dr. med., Ärztlicher Direktor, Abteilung für Unfallchirurgie und Orthopädische Chirurgie, Berufsgenossenschaftliche Unfallklinik Frankfurt am Main

Korrespondenzadresse

\section{Dr. med. Michael Kremer}

Abteilung für Unfallchirurgie und Orthopädische Chirurgie, Berufsgenossenschaftliche Unfallklinik Frankfurt am Main Friedberger Landstraße 430

60389 Frankfurt am Main

Michael.Kremer@BGU-Frankfurt.de

\section{Erstveröffentlichung}

Dieser Beitrag wurde erstveröffentlicht in: Günther KP, Hoffmann R, Hrsg. SOPs in der Orthopädie und Unfallchirurgie. Stuttgart: Thieme; 2017. 
[1] Boonen S, Adachi JD, Man Z et al. Treatment with denosumab reduces the incidence of new vertebral and hip fractures in postmenopausal women at high risk. J Clin Endocrinol Metab 2011; 96 (6): 1727-1736

[2] Duncan CP, Haddad FS. The Unified Classification System (UCS): improving our understanding of periprosthetic fractures. Bone Joint J 2014; 96-B: 713-716

[3] Ebraheim NA, Kelley LH, Liu X et al. Periprosthetic Distal Femur Fracture after Total Knee Arthroplasty: A Systematic Review. Orthop Surg 2015; 7 (4): 297-305

[4] Ha CW, Shon OJ, Lim SW, Park KH. Minimally invasive plate osteosynthesis for periprosthetic distal femoral fractures after total knee arthroplasty. Knee Surg Relat Res 2014; 26: 27-32

[5] Herrera DA, Kregor PJ, Cole PA et al. Treat- ment of acute distal femur fractures abo- ve a total knee arthroplasty: systematic review of 415 cases (1981-2006). Acta Orthop 2008; 79: 2227

[6] Lindahl H. Epidemiology of periprosthetic femur fracture around a total hip arthroplasty. Injury 2007; 38: 651-654

[7] Lindahl H, Malchau H, Odén A, Garellick G. Risk factors for failure after treatment of a periprosthetic fracture of the femur. J Bone Joint Surg Br 2006; 88 (1): 26-30

[8] Masri B, Meek R, Duncan C. Periprosthetic fracture evaluation and treatment. Clin Orthop Relat Res 2004; 420: 80-95

[9] Mittlmeier T, Stöckle U, Perka C et al. Periprothetische Frakturen nach Knietotalendoprothetik. Unfallchirurg 2005; 108: 481-496

[10] Ruchholtz S, Tomás ], Gebhard F et al. Periprosthetic fractures around the knee - the best way of treatment. Eur Orthop Traumatol 2013; 4: 93-102

Bibliografie

DOI https://doi.org/10.1055/a-0663-3357

Orthopädie und Unfallchirurgie up2date 2019; 14: 345-351

(c) Georg Thieme Verlag KG Stuttgart · New York

ISSN 1611-7859 